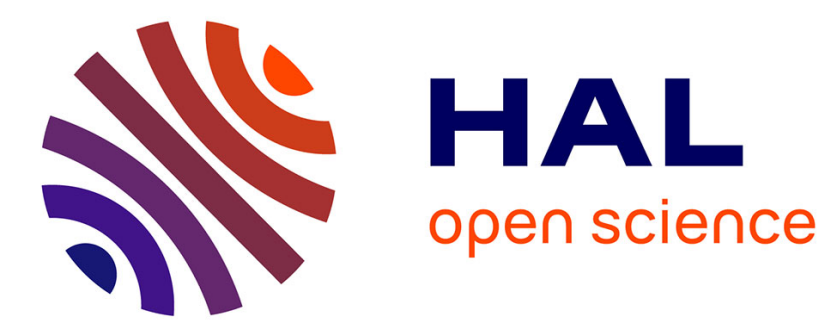

\title{
On the phase analysis of multi-carrier signals for high-precision fault detection by reflectometry
}

\author{
Esteban Cabanillas, Christophe Layer, Moussa Kafal
}

\section{To cite this version:}

Esteban Cabanillas, Christophe Layer, Moussa Kafal. On the phase analysis of multi-carrier signals for high-precision fault detection by reflectometry. 2017 IEEE SENSORS (ICSENS 2017), Oct 2017, Glasgow, United Kingdom. pp.8234182, 10.1109/ICSENS.2017.8234182 cea-01817858

\section{HAL Id: cea-01817858 https://hal-cea.archives-ouvertes.fr/cea-01817858}

Submitted on 18 Dec 2020

HAL is a multi-disciplinary open access archive for the deposit and dissemination of scientific research documents, whether they are published or not. The documents may come from teaching and research institutions in France or abroad, or from public or private research centers.
L'archive ouverte pluridisciplinaire HAL, est destinée au dépôt et à la diffusion de documents scientifiques de niveau recherche, publiés ou non, émanant des établissements d'enseignement et de recherche français ou étrangers, des laboratoires publics ou privés. 


\title{
On the Phase Analysis of Multi-Carrier Signals for High-Precision Fault Detection by Reflectometry
}

\author{
Esteban Cabanillas, Christophe Layer, Moussa Kafal \\ CEA, LIST, Laboratoire de Fiabilisation et d'Intégration des Capteurs \\ Nano-Innov, Bât. 862-PC172, 91191 Gif-sur-Yvette Cedex, France \\ \{esteban.cabanillas, christophe.layer, moussa.kafal\}@cea.fr
}

\begin{abstract}
Electronic systems are becoming always more complex and consequently more subject to defect. For safety, security and integrity reasons, wire diagnosis is crucial. The emerging of sensor networks and connected objects has created the need for embedded and non invasive fault diagnosis solutions. Actual systems rely on multi-carrier reflectometry to locate upcoming defects on wires, though their precision stays within the physical limits of their components, especially the sampling frequency of their analog parts. We propose a new approach combining multicarrier reflectometry and phase analysis to overcome this limit, in order to improve the precision of the localization of the defects on electrical wires. Based on an FPGA implementation, our novel method and the resulting system has proven a five-times better accuracy than state-of-the-art methods on the same platform.
\end{abstract}

Keywords-Reflectometry, phase analysis, Fourier transform, multi-carrier, defect diagnosis, high precision sensing.

\section{INTRODUCTION}

Due to their reliability, even in the wireless era, wiring cables are everywhere possible and still constitute the backbone of every electronic system, vehicle, aircraft or even larger structures. As various reflectometry methods have long been studied to estimate the wealth of electrical wires, they have reached a limit in terms of precision due to the physical capacities of their constituting components. However, not knowing the exact location of a fault causes larger non-recurring reparation costs and yields longer immobilization time of the failing object. Nowadays, the challenge has moved towards detecting faults at a higher precision without expensively improving almost every single item of the system, when even possible. In fact, embedded systems and distributed sensor networks aim at being cost-efficient while energy efficient and sufficiently performing. Embedded and distributed wire diagnosis systems rely basically on the analysis of the electrical waves echoed on impedance mismatches along the cable under test (CUT), as seen in Fig. 1. The principle of Time-Domain Reflectometry (TDR) is to inject a test signal $x(t)$ on the CUT, analyze the electrical behavior $y(t)$ and detect the potential reflections through a correlation $\Gamma_{x y}(t)$ with

$$
\Gamma_{x y}(t)=\int_{-\infty}^{\infty} y(t+\tau) \cdot s^{*}(t) d \tau
$$

To this aim, analog-to-digital converters are in charge of sampling the signal injections and reflections, but their accuracy is physically limited, and so is the spatial resolution $\delta_{l}$ with

$$
\delta_{l}=\frac{v_{g}}{2 f_{s}}=\frac{v_{g} \cdot T_{s}}{2} \quad \text { with } \quad f_{s}=T_{s}^{-1}
$$

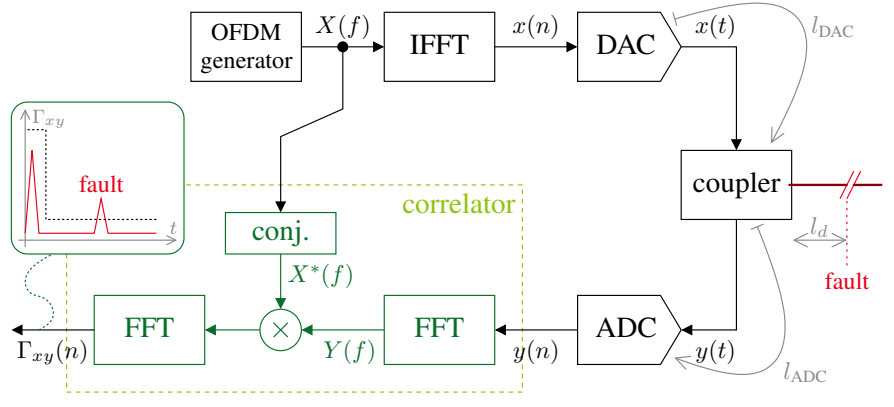

Fig. 1. Schematic diagram of a standard wire diagnosis system using a correlator between the injected signal $x(n)$ and the received signal $y(n)$ to detect the location of the fault.

where $f_{s}$ is the sampling frequency of the converters and $v_{g}$ the propagation speed on the wire. Existing methods use the periodicity of the injected signal to emulate a fake oversampling by periodically time-shifting the sampling point, but this method is costly, very slow, and totally jitter intolerant. Moreover, intermittent faults cannot be detected due to the too long acquisition time.

On the one hand, Frequency-Domain Reflectometry (FDR) relies on measuring the complex response of a network for separated frequencies in the desired bandwidth. Similar methods such as Phase Detection FDR (PDFDR) [1] and Mixed-Signal Reflectometry (MSR) [2] have also been studied [3], [4]. In practice, they yield similar performance as TDR. On the other hand, standard TDR [5] is based on a Dirac-pulse to recognize impedance discontinuities. The frequency spectrum of the injected signal is hence infinitely large, preventing this method from being suitable for embedded systems and distributed sensors networks. In order not to impact the functionality of a system during live-diagnosis, specific methods based on OFDM (Orthogonal Frequency Division Multiplexing) such as MCTDR (Multi-Carrier TDR) [6], OMTDR (Orthogonal Multi-tone TDR) [7] and modulated variants [8] shall be preferred, as they provide a complete control of the spectrum of the injected signal which shall not interfere with the system under test. This technique basically divides the channel bandwidth into many sub-carriers independently modulated, hence providing a better spectral efficiency.

In this paper, an approach to fusion the multi-carrier signals used by OFDM techniques with frequency domain analysis will be presented. Fortunately, this will produce a low complexity system architecture along with high precision fault measurements. Besides, an innovative solution based 
on frequency analysis from multi-carrier signals will also be described. The implementation of this method is treated in section III. Measurement results using an FPGA platform and external analog converters are presented in section IV.

\section{PROPAGATION OF ELECTROMAGNETIC SIGNALS AND ITS PRINCIPLE FOR FAULT DETECTION}

The propagation of an electromagnetic signal through a cable of length $l$ is defined in the frequency domain as

$$
Y(f)=X(f) H(f) e^{-j \phi(l, f)} \quad \text { with } \quad \phi(l, f)=2 \pi f \frac{l}{v_{g}}
$$

where $X(f)$ and $Y(f)$ are the frequency-domain incident and reflected signals respectively, and $H(f)$ the attenuation function, and $\phi(l, f)$ is the phase function, as defined in [8]. In the case of a system detecting and locating faults in a CUT by reflectometry, $l$ is twice the distance to fault. This distance can be then obtained by controlling the frequency $f$ of the injected signal with an a-priori knowledge of $v_{g}$.

As the arctangent function returns a value in $[-\pi, \pi]$, the phase of the reflected signal $Y(f)$ propagating over a CUT of length $l$ for a given frequency $f_{i}$ can be equal or greater than that of a frequency $f_{j}$, even if $f_{i}<f_{j}$. Consequently, the sought-after length $l^{\prime}$ is not the same for $f_{i}$ and $f_{j}$ with a potentially wrong estimation for both cases. Getting a reliable estimation of the fault location on the CUT is possible by using multi-carrier signals. The multi-carrier signal spectrum $X(f)$ can be defined as a group of carriers with different frequencies $f_{n}$ given by the following amplitude and phase functions:

$$
\left|X\left(f_{n}\right)\right|=1 \forall f_{n} \quad \text { and } \quad \phi\left(f_{n}\right)=\phi_{n}
$$

Accordingly, the fault's location is relatively obtained from the difference of the phases of two adjacent carriers:

$$
\phi\left(l, f_{n+1}\right)-\phi\left(l, f_{n}\right)=2 \pi f_{n+1} \frac{l}{v_{g}}-2 \pi f_{n} \frac{l}{v_{g}},
$$

where the length $l^{\prime}$ we are looking for becomes:

$$
l^{\prime}=\frac{v_{g}}{2 \pi} \frac{\phi\left(l, f_{n+1}\right)-\phi\left(l, f_{n}\right)}{f_{n+1}-f_{n}}=\frac{v_{g}}{2 \pi} \frac{\Delta_{\phi}}{\Delta_{f}}
$$

It is noteworthy that choosing the right frequency spacing between carriers $\Delta_{f}$ is fundamental to avoid getting a phase difference $\Delta_{\phi}>2 \pi$. The maximum value of $\Delta_{f}$ can be obtained by fixing a maximum fault distance:

$$
\Delta_{\phi}=2 \pi \Delta_{f} \frac{l_{\max }}{v_{g}}<2 \pi \quad \Longrightarrow \quad \Delta_{f}<\frac{v_{g}}{l_{\max }}
$$

\section{NOVEL WIRE FAULT DETECTION SYSTEM BASED ON THE ANALYSIS OF THE PHASE OF THE REFLECTION}

The system model describing the elements performing fault detection from multi-carrier phase analysis is presented in Fig. 2. As for OFDM, the time-domain multi-carrier signal is generated by a multi-carrier generator which sets the amplitude and phase of every single carrier. An IFFT block then transforms the multi-carrier spectrum from the frequency domain into the time domain. Besides, a DAC is coupled to an ADC, hence traveling towards the CUT. In our case, the novel system is simplified by using a sole FFT block that feeds a phase computing block Atan $(\cdot)$, relying on standard computing

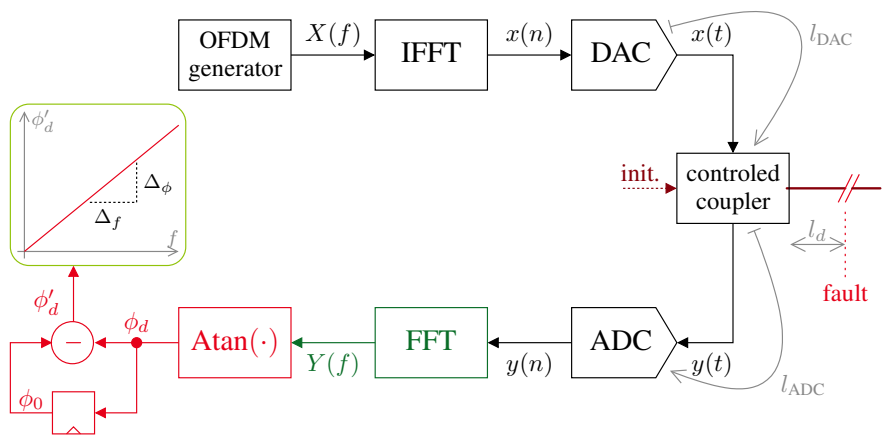

Fig. 2. Schematic diagram of the novel wire diagnosis system using only an FFT to analyze the phase of the reflected reflectometry signal and locate a fault using the variation of the phase versus the variation of the frequency.

methods for trigonometric functions such as described in [9]. Note that integer arithmetic is precise enough in the area of fault detection and diagnosis. The maximal value of $\Delta_{f}$, defined in (7), can be set by choosing the right number of carriers $N$ and the DAC sampling frequency $f_{s}$.

Locating a fault using the system presented in Fig. 2 is accomplished in three steps. The first step consists of injecting the multi-carrier signal when the cable is not coupled to the reflectometry device. Coupling and decoupling the cable can be done in an autonomous mode by implementing a switch. The phase $\phi_{0}\left(l, f_{n}\right)$ for the carrier of frequency $f_{n}$ with no wire coupled is defined as:

$$
\begin{aligned}
\phi_{0}\left(l, f_{n}\right) & =\phi_{n}+\phi\left(l_{\mathrm{DAC}}+l_{\mathrm{ADC}}, f_{n}\right) \\
& =\phi_{n}+2 \pi f_{n} \frac{l_{\mathrm{DAC}}+l_{\mathrm{ADC}}}{v_{g}}
\end{aligned}
$$

where $\phi_{n}$ is the initial phase of the carrier of frequency $f_{n}$ as given by (4), $l_{\mathrm{DAC}}$ is the path length between the DAC and the coupler, and $l_{\mathrm{ADC}}$ is the path length between the coupler and the ADC. Each value $\phi_{0}\left(l, f_{n}\right)$ is stored as a reference measurement in the register in front of the subtracter. In the second step, the CUT is bounded to the coupler. The phase of the carrier with frequency $f_{n}$ for a cable with a fault at a distance $l_{d}$ can be expressed as:

$$
\begin{aligned}
\phi_{d}\left(l, f_{n}\right) & =\phi_{n}+\phi\left(l_{\mathrm{DAC}}+l_{\mathrm{ADC}}, f_{n}\right)+\phi\left(2 l_{d}, f_{n}\right) \\
& =\phi_{n}+2 \pi f_{n} \frac{l_{\mathrm{DAC}}+l_{\mathrm{ADC}}}{v_{g}}+2 \pi f_{n} \frac{2 l_{d}}{v_{g}}
\end{aligned}
$$

The last steps consists in subtracting from $\phi_{d}\left(l, f_{n}\right)$ the phases stored on the registering block in front of the subtracter:

$$
\phi_{d}^{\prime}\left(l, f_{n}\right)=\phi_{0}\left(l, f_{n}\right)-\phi_{d}\left(l, f_{n}\right)=2 \pi f_{n} \frac{2 l_{d}}{v_{g}}
$$

It is noteworthy that the part of the phase induced by the distances $l_{\mathrm{DAC}}$ and $l_{\mathrm{ADC}}$ has been avoided by applying a baselining approach where the a reference measure is subtracted from the CUT measure. Accordingly, the only unknown parameter is $l_{d}$ which can be estimated from (6) by considering two adjacent frequencies. Furthermore, taking several adjacent carriers permits a linear regression which yields a better precision in estimating the location of the fault. As a result, the phase value must be a continuous function, not bounded within $[-\pi, \pi]$. Such a function is obtained by cumulating the 


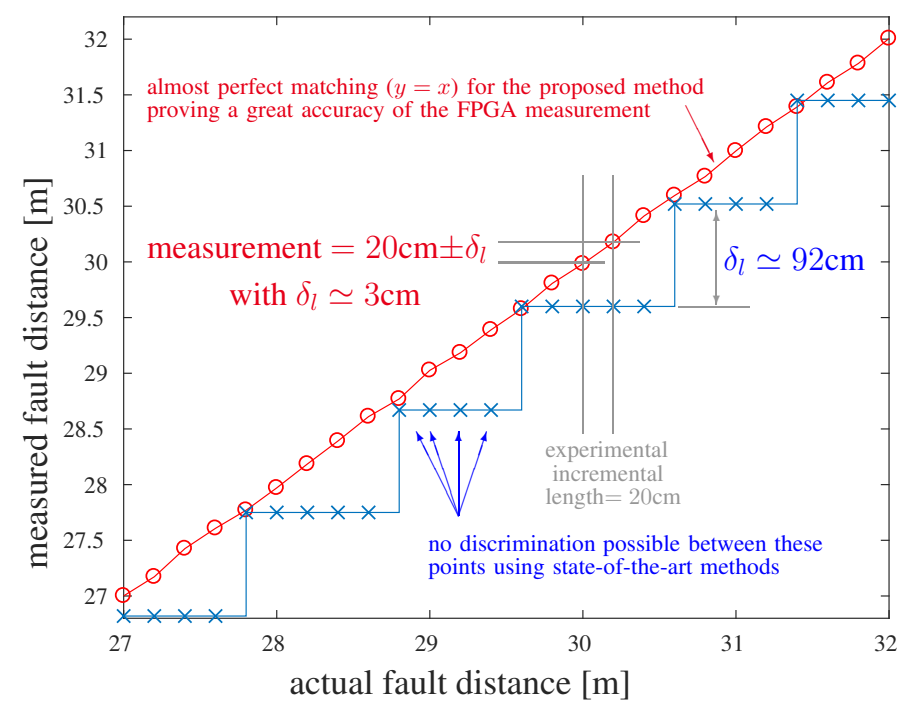

Fig. 3. Measurement of the distance to the fault on a hardware platform versus the actual fault distance for a system performing state-of-the-art correlationbased reflectometry (blue) and another system performing our new localization method relying directly on the phase of the reflected signal (red).

phases values of previous frequencies. Thus, the phase value for a given frequency $f_{n+M}$ and a given fault location $l$ is

$$
\phi_{c}^{\prime}\left(l, f_{n+M}\right)=\sum_{i=0}^{M} \phi_{d}^{\prime}\left(l, f_{n+i}\right)
$$

The presented method has several advantages in an embedded system scenario when compared to traditional reflectometry based on OFDM signals. As a matter of fact, it is capable of cutting some frequency bands without any degradation in the diagnosis of the CUT. The autocorrelation function of an OFDM signal is strongly degraded when one or several carriers are attenuated, and so is the sensitivity and the precision in MCTDR or OMTDR. This is not an issue for the phase analysis since the user chooses the first and last frequencies used to estimate the location of the fault.

\section{MEASUREMENTS AND ANALYSIS}

The system presented on Fig. 2 has been implemented on an FPGA board driving a 10-bits DAC and a 10-bit ADC. Both converters are tightly coupled and connected to the CUT. The DAC and ADC sampling frequency is $100 \mathrm{MHz}$. The multi-carrier signal is made from an OFDM modulator which generates 128 carriers. As specified by the OFDM method, these carriers are equally distributed in the interval from 0 to $100 \mathrm{MHz}$. The frequency spacing between carriers is around $787 \mathrm{kHz}$, which permits to test a cable whose length is shorter than $250 \mathrm{~m}$ with propagation speed $v_{g}$ of $1.85 \cdot 10^{8} \mathrm{~m} / \mathrm{s}$. It is important to note that this propagation speed was used in the experimental validation.

The goal of the measurements in this section is to locate a defect in the CUT by using two reflectometry methods, both injecting the same multi-carrier signal. The first method is the well known correlation method, which performs (1) between the reference and the acquisition to detect and locate a fault in the wire. The second method is the one introduced in Sec. II, in which the location of the fault is accomplished by analyzing the phase of each carrier of the received signal. Several acquisitions have been made for different open-circuit distances in the CUT with respect to coupling point. The results of the measurements are illustrated in Fig. 3 where each point represents an acquisition on the FPGA. Effectively, the length of the CUT has been reduced by steps of $20 \mathrm{~cm}$ at each acquisition. As seen on Fig. 3, the resolution of the estimation obtained by traditional correlation-based techniques, i.e., the blue line, is of $\delta_{l}=92 \mathrm{~cm}$. The resolution described in (2) is now greatly improved with the novel technique proposed in this paper in Sec. III, represented by the red line. As noted on Fig. 3, there is no actual discontinuity between two adjacent points using our novel method. The granularity reveals the repetition steps during our test-bench, where the faults have been produced with a spacing of $20 \mathrm{~cm}$. The maximum measured error at each point was $\delta_{l}=3 \mathrm{~cm}$. The actual spacial resolution of our new method is finally a function of the digital quantification of the DAC and the ADC, and of the experimentally coupled noise.

\section{Conclusion}

As the characteristics of a defect in a wire are specified in the phase information, we have proposed a novel method of wire fault diagnosis based on the analysis of the evolution of the phase of multi-carrier signals. The needed material necessary to implement this method is similar to state-of-theart methods, which allows its integration into low cost and embedded hardware systems. The presented system permits to localize and characterize defects with a precision not reachable through standard reflectometry techniques, limited by DAC and ADC performances. In effect, a faulty cable has been tested with both traditional correlation method and our new phasedetection method. While the error of the traditional method has a the spacial resolution of $92 \mathrm{~cm}$ in our test-bench, our proposed technique presents an error of around only $3 \mathrm{~cm}$ to the actual measurements of the location of the fault.

\section{REFERENCES}

[1] C. Furse, Y. Chung, R. Dangol, et al. "Frequency-Domain Reflectometry for On-Board Testing of Aging Aircraft Wiring," in IEEE Trans. on Electromagnetic Compatibility, vol. 45, no. 2, pp. 306-315, 2003.

[2] P. Tsai, C. Lo, Y. Chung Chung and C. Furse, "Mixed-Signal Reflectometer for Location of Faults on Aging Wiring," in IEEE Sensors Journal, vol. 5, no. 6, pp. 1479-1482, 2005.

[3] S. Naik, C. Furse, and B. Farhang-Boroujeny, "Multicarrier Reflectometry," Sensors Journal, IEEE, vol. 6, no. 3, pp. 812-818, 2006.

[4] P. Smith, C. Furse, and J. Gunther, "Analysis of Spread Spectrum Time Domain Reflectometry for Wire Fault Location," Sensors Journal, IEEE, vol. 5, no. 6, pp. 1469-1478, 2005.

[5] T.-W. Pan, C.-W. Hsue, and J.-F. Huang, "Time-Domain Reflectometry using Arbitrary Incident Waveforms," IEEE Trans. Microw. Theory Tech., vol. 50, no. 11, pp. 25582563, 2002.

[6] A. Lelong, M. Olivas, "Online Wire Diagnosis using Multi-Carrier Time Domain Reflectometry for Fault Location", in Proc. IEEE Sensors Conference, pp. 751-754, 2009.

[7] W. Ben Hassen, et al. "OMTDR using BER Estimation for Ambiguities Cancellation in Ramified Networks Diagnosis," in Proc. IEEE 8th Int. Conf. Intell. Sensors, Sensor Netw. Inf. Process., pp. 414-419, 2013.

[8] C. Layer, E. Cabanillas, and J. Benoit, "Using Quadrature Modulation for Precise Fault Location over Wired Communication Channels," in Proc. IEEE Int. Conf. New Circuits and Systems, 2017.

[9] R. Andraka, "A Survey of CORDIC Algorithms for FPGA based Computers", in Proc. ACM/SIGDA 6th International Symposium on Field Programmable Gate Arrays, pp. 191-200, 1998. 